\begin{tabular}{|c|l|}
\hline Title & Phonon group velocity and thermal conduction in superlattices \\
\hline Author(s) & Tamura, Shin-ichiro; Tanaka, Y ukihiro; Maris, Humphrey J. \\
\hline Citation & $\begin{array}{l}\text { PHY SICAL REVIEW B, 60(4), 2627-2630 } \\
\text { https://doi.org/L0.1103/PhysRevB.60.2627 }\end{array}$ \\
\hline Issue Date & 1999-07-15 \\
\hline Doc URL & http://hdl.handle.net/2115/5917 \\
\hline Rights & Copyright $\odot 1999$ A merican Physical Society \\
\hline Type & article \\
\hline File Information & PRB60-4.pdf \\
\hline
\end{tabular}

Instructions for use 


\title{
Phonon group velocity and thermal conduction in superlattices
}

\author{
Shin-ichiro Tamura and Yukihiro Tanaka \\ Department of Applied Physics, Hokkaido University, Sapporo 060-8628, Japan \\ Humphrey J. Maris \\ Department of Physics, Brown University, Providence, Rhode Island 02912
}

(Received 25 January 1999)

\begin{abstract}
With the use of a face-centered cubic model of lattice dynamics we calculate the group velocity of acoustic phonons in the growth direction of periodic superlattices. Comparing with the case of bulk solids, this component of the phonon group velocity is reduced due to the flattening of the dispersion curves associated with Brillouin-zone folding. The results are used to estimate semiquantitatively the effects on the lattice thermal conductivity in $\mathrm{Si} / \mathrm{Ge}$ and GaAs/AlAs superlattices. For a Si/Ge superlattice an order of magnitude reduction is predicted in the ratio of superlattice thermal conductivity to phonon relaxation time [consistent with the results of P. Hyldgaard and G. D. Mahan, Phys. Rev. B 56, 10754 (1997)]. For a GaAs/AlAs superlattice the corresponding reduction is rather small, i.e., a factor of 2-3. These effects are larger for the superlattices with larger unit period, contrary to the recent measurements of thermal conductivity in superlattices. [S0163-1829(99)05028-6]
\end{abstract}

The propagation and scattering of acoustic phonons in a multilayered structure are of both fundamental and practical interest. The basic propagation characteristics of phonons in superlattices have been extensively studied, both theoretically and experimentally. ${ }^{1}$ Recently, thermal transport in semiconducting $^{2-16}$ multilayered structures has attracted much attention due to potential application to quantum-well lasers and thermoelectric devices. ${ }^{17}$

The thermal conductivities both parallel $\mathrm{e}^{2,5,6}$ and perpendicular $^{3,10-13}$ to the interfaces of semiconductor superlattices have been measured by several groups, and significant reductions compared with the values in bulk materials are reported. The thermal conductivity measured in the inplane direction is smaller than the average conductivity of the bulk constituents and decreases with decreasing superlattice period. Chen, ${ }^{9}$ and Hyldgaard and Mahan ${ }^{14}$ suggested that diffuse superlattice-phonon interface scattering is the key factor in explaining the thermal-conductivity reduction in the direction parallel to the layer interfaces.

A more dramatic reduction has been found in the thermal conductivity in the growth direction for both GaAs/AlAs and $\mathrm{Si} / \mathrm{Ge}$ superlattices. ${ }^{3,10-13}$ Over a wide range of temperatures (80-330 K), Capinski and co-workers ${ }^{10-12}$ made measurements with GaAs/AlAs samples of various repeat distances. At $300 \mathrm{~K}$ they observed that the thermal conductivity is three times to an order of magnitude less than that of bulk GaAs, depending on the repeat distance. Lee et al. ${ }^{13}$ measured a series of $\mathrm{Si} / \mathrm{Ge}$ superlattices and found that the thermal conductivity of their samples fell below the conductivity of SiGe alloys, but above the conductivity of $a$-Si. Again, at high temperatures, for some of their samples, there was over an order of magnitude decrease compared to the average of the conductivity of the bulk silicon and germanium.

Chen and Neagu ${ }^{8}$ and $\mathrm{Chen}^{9}$ attributed the large reduction of the thermal conductivity in GaAs/AlAs and Si/Ge superlattices to diffuse interface scattering or dislocation scattering of phonons, depending on the thickness of bilayers. An alternative approach was taken by Hyldgaard and Mahan. ${ }^{15}$ They considered the possibility that the thermal conductivity in a superlattice is reduced because the phonon group velocity is decreased relative to its value in the bulk constituents. They employed a simple-cubic lattice model, and predicted that due to this effect there should be an order of magnitude reduction in the thermal conductivity of a $(2 \times 2)-\mathrm{Si} / \mathrm{Ge}$ superlattice. However, they have not shown how this reduction changes as the length of the unit period is varied. In the present work we employ a face-centered cubic (fcc) lattice dynamics model, and study the group-velocity effect more systematically than Hyldgaard and Mahan. ${ }^{15}$ We show for $\mathrm{Si} / \mathrm{Ge}$ superlattices how the reduction in the conductivity changes with the repeat distance of the superlattice. We also make similar calculations with model parameters appropriate for GaAs/AlAs superlattices to consider if the experimental results of Capinski and co-workers ${ }^{10-12}$ can be explained by the reduction in phonon group velocity.

Here we note that the lattice thermal conductivity $\kappa$ at a temperature $T$ is given by the formula

$$
\begin{gathered}
\kappa=\sum_{\lambda} \kappa_{\lambda}, \\
\kappa_{\lambda}=\tau_{\lambda} C_{p h}\left(\omega_{\lambda}\right) \mathrm{v}_{\lambda, z}^{2}=\tau_{\lambda} C_{p h}\left(\omega_{\lambda}\right) \mathrm{v}_{\lambda}^{2} \cos ^{2} \theta_{\lambda}, \\
C_{p h}(\omega)=\frac{(\hbar \omega)^{2}}{k_{B} T^{2}} \frac{\exp \left(\hbar \omega / k_{B} T\right)}{\left[\exp \left(\hbar \omega / k_{B} T\right)-1\right]^{2}},
\end{gathered}
$$

where $\lambda$ is a set of quantum numbers specifying a phonon state, which we choose for superlattice phonons as $\lambda$ $=\left(\mathbf{k}_{\|}, q, j\right)$ with $\mathbf{k}_{\|}$the wave vector parallel to the layer interfaces, $q$ the wave number in the growth direction, and $j$ the index specifying both the phonon mode and frequency band. Also in Eq. (2) $\tau$ is the relaxation time of phonons, $\omega$ is the angular frequency, $\mathrm{v}$ is the magnitude of the phonon group 


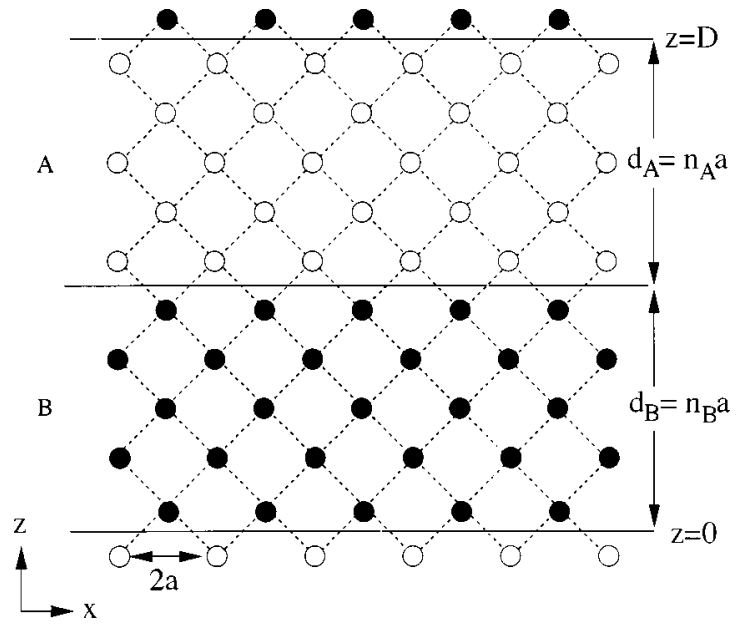

FIG. 1. Structure of a unit period in the $\left(n_{A} \times n_{B}\right)$ superlattice consisting of two fcc lattices with different masses.

velocity, and $\theta$ is the angle between the group velocity and the direction of the heat flow. In the experiments the thermal conductivity normal to the interfaces ( $z$ direction) has been measured, so we write $\mathrm{v}_{\lambda} \cos \theta_{\lambda}=\mathrm{v}_{\lambda, z}$ in Eq. (2).

Now we consider a periodic superlattice whose unit period consists of two fcc lattices (lattice $A$ and lattice $B$ ) divided by an interface between two adjacent (001) planes of atoms parallel to the $x-y$ plane (see Fig. 1). In lattice $A(B)$, atoms of mass $M_{A}\left(M_{B}\right)$ are connected to their 12 nearest neighbors by springs of stiffness $K$, i.e., we assume the same magnitude of atomic force constants for $A-A, B-B$, and $A-B$ pairs. The spacing between the nearest neighbors is taken to be $\sqrt{2} a$. Thus, the thickness of sublattice $A(B)$ is $d_{A}$ $=n_{A} a \quad\left(d_{B}=n_{B} a\right)$, where $n_{A} \quad\left(n_{B}\right)$ is the number of atomic layers and the length of unit period is $D=d_{A}+d_{B}$.

We write $\mathbf{u}_{l m n}$ to be the displacement of an atom from an equilibrium position $\mathbf{r}_{l m n}=\left(\mathbf{x}_{l m}, z_{n}\right)=(l a, m a, n a)$ in the $N$ th period of the lattice. The displacement vector that satisfies Bloch's theorem required for the periodicity in the $z$ direction takes the form

$$
\mathbf{u}_{l m n}=\mathbf{u}_{n}^{\tilde{e x p}}\left[i\left(\mathbf{k}_{\|} \cdot \mathbf{x}_{l m}+q N D-\omega t\right)\right],
$$

for $(N-1) n_{A B} \leqslant n<N n_{A B}$, where $n=N n_{A B}+\tilde{n}$ with $n_{A B}$ $=n_{A}+n_{B}$ the number of atoms in $z$ direction in the unit period, and $1 \leqslant \tilde{n} \leqslant n_{A B}$. In Eq. (4) $\mathbf{k}_{\|}=\left(k_{x}, k_{y}\right)$ is common to lattices $A$ and $B$, and $q$ is the Bloch wave number. The equation of motion satisfied by $\mathbf{u}_{l m n}$ is ${ }^{18}$

$$
\begin{aligned}
M_{n} \ddot{u}_{l m n}^{x}= & \frac{K}{2}\left(u_{l+1, m+1, n}^{x}+u_{l+1, m, n+1}^{x}+u_{l+1, m-1, n}^{x}\right. \\
& +u_{l+1, m, n-1}^{x}+u_{l-1, m+1, n}^{x}+u_{l-1, m, n+1}^{x}+u_{l-1, m-1, n}^{x} \\
& +u_{l-1, m, n-1}^{x}-8 u_{l m n}^{x}+u_{l+1, m+1, n}^{y}-u_{l+1, m-1, n}^{y} \\
& +u_{l-1, m-1, n}^{y}-u_{l-1, m+1, n}^{y}+u_{l+1, m, n+1}^{z}-u_{l+1, m, n-1}^{z} \\
& \left.+u_{l-1, m, n-1}^{z}-u_{l-1, m, n+1}^{z}\right),
\end{aligned}
$$

where $M_{n}=M_{A}$ or $M_{B}$ depending on the site $n$, and the equations of motion for $\ddot{u}_{l m n}^{y}$ and $\ddot{u}_{l m n}^{z}$ are obtained by a
TABLE I. Comparison between the calculated (calc) and experimental (expt) sound velocities of the bulk longitudinal $(l)$ and transverse $(t)$ modes in the [100] direction (in units of $10^{5} \mathrm{~cm} / \mathrm{s}$ ).

\begin{tabular}{lllll}
\hline \hline $\mathrm{I}$ & $\mathrm{v}_{l}^{\text {calc }}$ & $\mathrm{v}_{l}^{\text {expt }}$ & $\mathrm{v}_{t}^{\text {calc }}$ & $\mathrm{v}_{t}^{\text {expt }}$ \\
\hline $\mathrm{Si}$ & 8.48 & 8.34 & 5.86 & 5.90 \\
$\mathrm{Ge}$ & 4.97 & 5.18 & 3.58 & 3.67 \\
$\mathrm{GaAs}$ & 4.71 & 4.71 & 3.33 & 3.33 \\
$\mathrm{AlAs}$ & 5.65 & 5.61 & 3.96 & 3.96 \\
\hline \hline
\end{tabular}

cyclic permutation of the subscripts and the superscript in Eq. (5). These equations determine $3 n_{A B}$ frequencies $\omega$ as the functions of $\mathbf{k}_{\|}$and $q(0 \leqslant q \leqslant \pi / D)$.

We apply the above formulas to $\mathrm{Si} / \mathrm{Ge}$ and GaAs/AlAs superlattices with different length of a unit period. Hence, first we determine the parameters involved in the fcc model so that the model may best approximate the constituent materials of the superlattices. Here we note that silicon and germanium take diamond structures where a unit cell consists of two identical atoms. So, in applying the fcc model we assign $M_{A}\left(=2 \times M_{\mathrm{Si}}\right)=9.32 \times 10^{-23} \mathrm{~g}$ and $M_{B} \quad(=2$ $\left.\times M_{\mathrm{Ge}}\right)=2.41 \times 10^{-22} \mathrm{~g}$ for a $\mathrm{Si} / \mathrm{Ge}$ superlattice, where $M_{\mathrm{Si}}$ and $M_{\mathrm{Ge}}$ are the atomic masses of silicon and germanium, respectively. We also assume the lattice constant $\tilde{a}=2 a$ $=5.54 \times 10^{-8} \mathrm{~cm}$, which is the mean value of the lattice constants of bulk silicon and germanium. The force constant is taken to be $K=4.22 \times 10^{4} \mathrm{~g} \mathrm{~s}^{-2}$. These parameters give the bulk sound velocities in the [100] direction tabulated in Table I.

For a GaAs/AlAs superlattice we also consider only acoustic vibrations and replace two atoms in a unit cell of each material with a single atom in the fcc lattice. Thus, the chosen values are $M_{A}\left(=M_{\mathrm{AlAs}}\right)=1.69 \times 10^{-22} \mathrm{~g}$ and $M_{B}\left(=M_{\mathrm{GaAs}}\right)=2.40 \times 10^{-22} \mathrm{~g}$ and the lattice constant $\tilde{a}$ $=2 a=5.64 \times 10^{-8} \mathrm{~cm}$ and $K=3.35 \times 10^{4} \mathrm{~g} \mathrm{~s}^{-2}$. The calculated sound velocities are also given in Table I.

We have shown in Fig. 2 the dispersion curves in the $(5 \times 5)-\mathrm{Si} / \mathrm{Ge}$ and $(5 \times 5)-\mathrm{AlAs} / \mathrm{GaAs}$ superlattices for
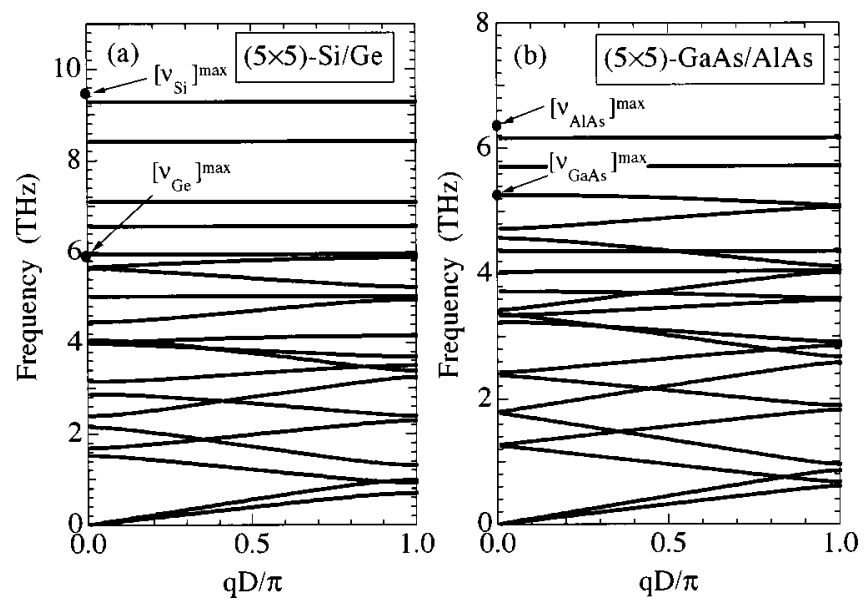

FIG. 2. Dispersion relations of phonons in the growth direction of (a) a $(5 \times 5)-\mathrm{Si} / \mathrm{Ge}$ superlattice and (b) a $(5 \times 5)-\mathrm{AlAs} / \mathrm{GaAs}$ superlattice. Frequency $\left[\nu_{I}\right]^{\max }$ (with $I=\mathrm{Si}, \mathrm{Ge}, \mathrm{GaAs}$, and AlAs) indicates the maximum frequency of the bulk $I$ material calculated by the fcc model. 

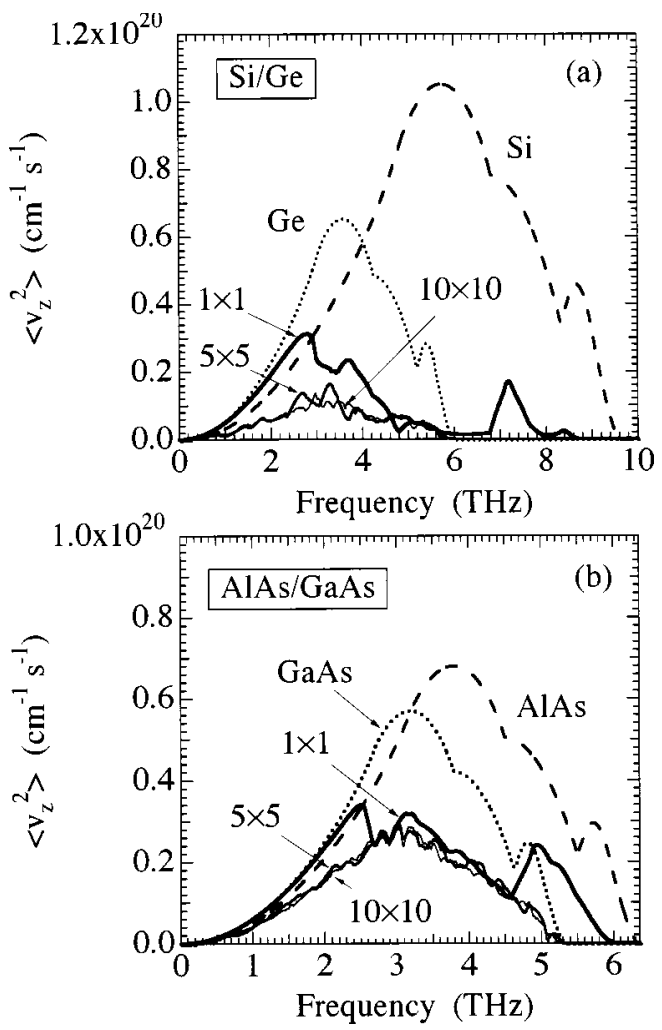

FIG. 3. Densities of states weighted by the squared $z$ component (the component in the growth direction) of the group velocity. (a) $(n \times n)-\mathrm{Si} / \mathrm{Ge}$ superlattices with $n=1,5$, and 10, and (b) $(n \times n)$-AlAs/GaAs superlattice with $n=1,5$, and 10. For comparison the same quantities of bulk solids are also shown by dashed lines.

phonons propagating in the growth direction. The frequency gaps at the center and boundary of the folded Brillouin zone are larger for the $\mathrm{Si} / \mathrm{Ge}$ superlattice because of the larger mass differences of the constituent atoms, and hence the reductions of phonon group velocity towards the zone center and boundary are also larger in the $\mathrm{Si} / \mathrm{Ge}$ superlattice. Here we note that the calculated sound velocities coincide very well with the experimental values but the fcc model predicts the maximum phonon frequencies at the zone boundary $\left(\omega_{A}^{\max }=2 \pi \nu_{A}^{\max }=\left(8 K / M_{A}\right)^{1 / 2}\right)$, which are about $20 \%$ smaller than the measured values of the bulk materials considered.

In order to check our computer code we have calculated the lattice specific heat. As expected, the specific heats per atom of superlattices take values in between those of the constituent bulk materials at low temperatures, and approach the universal classical value of $3 k_{B}$ in the high-temperature limit.

We show in Figs. 3(a) and 3(b) the frequency dependence of the density of states (DOS) weighted by the square of the group velocity perpendicular to the interfaces, defined by

$$
\begin{aligned}
\left\langle\mathrm{v}_{z}^{2}(\omega)\right\rangle & \equiv \frac{1}{V} \sum_{\lambda} \delta\left(\omega-\omega_{\lambda}\right) \mathrm{v}_{\lambda, z}^{2} \\
& =\left.\frac{1}{(2 \pi)^{3}} \sum_{j} \int \frac{d S_{\lambda}}{\mathrm{v}_{\lambda}} \mathrm{v}_{\lambda, z}^{2}\right|_{\omega_{\lambda}=\omega},
\end{aligned}
$$
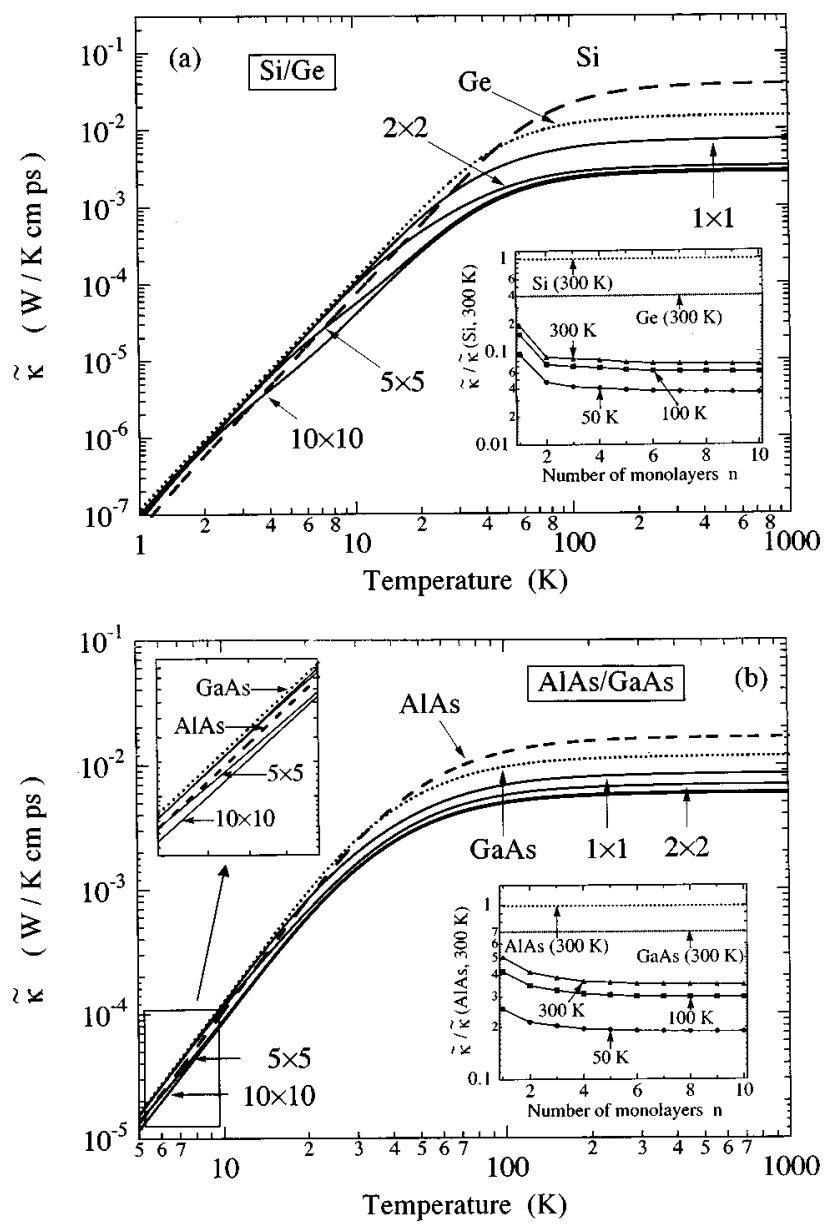

FIG. 4. The ratio $\tilde{\kappa}$ of thermal conductivity to phonon relaxation time. (a) $(n \times n)-\mathrm{Si} / \mathrm{Ge}$ superlattices with $n=1,2,5$, and 10, and (b) $(n \times n)$-AlAs/GaAs superlattices with $n=1,2,5$, and 10 . The corresponding values of the bulk solids are shown by dashed lines. Insets show the variation of $\tilde{\kappa}$ [normalized by the values of (a) bulk silicon and (b) bulk AlAs at $T=300 \mathrm{~K}$ ] with the number of monolayers $n$ in a unit cell. The results at $T=50,100$, and $300 \mathrm{~K}$ are plotted.

where $\mathrm{v}_{\lambda, z}=d \omega_{\lambda} / d q$, and $d S_{\lambda}$ is an element of a constantfrequency surface in the Brillouin zone.

In the low-frequency region the calculated weighted DOS for an $(n \times n)$-superlattice takes values between those of the bulk solids, but it is suppressed considerably at high frequencies due to the flattening of the phonon dispersion curves caused by the zone folding effects. As the number of monolayers $n$ in a unit period increases, the weighted DOS starts to deviate from the $\omega^{2}$ dependence at lower frequencies. We find that the weighted DOS is essentially the same for all values of $n$ larger than about 10 . There exists no contribution to this quantity from the frequencies higher than the maximum frequency $\omega_{B}^{\max }$ of the bulk solid of the heavier constituent. This is because the phonons in this frequency range are well localized inside the lighter layer $(A)$ of the unit period and have no effect on the heat conduction in superlattices.

Now we consider the ratio $\tilde{\kappa}$ of the thermal conductivity to phonon relaxation time defined by 


$$
\tilde{\kappa} \equiv \sum_{\lambda} \kappa_{\lambda} / \tau_{\lambda}=\sum_{\lambda} C_{p h}\left(\omega_{\lambda}\right) v_{\lambda, z}^{2} .
$$

The temperature dependences of this ratio (per volume) are shown in Figs. 4(a) and 4(b) for the $\mathrm{Si} / \mathrm{Ge}$ and AlAs/GaAs superlattices, respectively. The magnitude of $\tilde{\kappa}$ for the $(2 \times 2)-\mathrm{Si} / \mathrm{Ge}$ superlattice relative to those for the bulk silicon and germanium is very similar to the result by Hyldgaard and Mahan, ${ }^{15}$ i.e., there is an order of magnitude reduction at high temperatures. However, at $T=1000 \mathrm{~K}$ the absolute magnitude for the $(2 \times 2)$-Si/Ge superlattice we obtained is about $40 \%$ of their result. This should be due to the fact that different lattice dynamics models are used to evaluate $\tilde{\kappa}$. Although a large reduction of $\tilde{\kappa}$ is found for the $\mathrm{Si} / \mathrm{Ge}$ superlattices, the reduction in $\tilde{\kappa}$ for AlAs/GaAs superlattices is modest. At room temperatures $\tilde{\kappa}$ is about $1 / 2$ and $1 / 3$ of the corresponding values for the bulk GaAs and AlAs, respectively.

As the number of atomic layers $n$ in a unit period increases, $\tilde{\kappa}$ decreases, but it saturates for $n>10$ as shown in the insets. This $n$ dependence common to the $\mathrm{Si} / \mathrm{Ge}$ and AlAs/GaAs superlattices is contrary to the experimental results, i.e., the thermal conductivity of $\mathrm{AlAs} / \mathrm{GaAs}$ superlattices at room temperatures is reduced as the number $n$ is decreased. Also we see that at very low temperatures $\tilde{\kappa}$ is found between the curves of bulk solids irrespective of $n$, but it starts to deviate from the bulk lines as temperature in- creases. The temperature at which this deviation starts corresponds roughly to the frequency of the lowest phonon stop band in the dispersion relations.

To summarize, based on the fcc lattice model, we have calculated the phonon group velocities and estimated their contributions to the lattice thermal conductivity in $\mathrm{Si} / \mathrm{Ge}$ and $\mathrm{AlAs} / \mathrm{GaAs}$ superlattices in the growth direction. We found that the reduction of the group velocity near the folded Brillouin-zone center and edges yields a reduction in the thermal conductivity that is less than that found experimentally for AlAs/GaAs superlattices. This implies that while the phonon group velocity makes a very significant contribution to the reduction of the thermal conductivity in superlattices, other mechanisms must also play an important role. Possible effects other than phonon focusing ${ }^{19}$ include the change in the rate of anharmonic scattering, especially due to miniumklapp scattering, ${ }^{20}$ isotope scattering, ${ }^{21}$ and also scattering arising from the roughness of the layer interfaces.

Finally, we note that the reduction of the thermal conductivity in the microstructures such as dielectric quantum wires has also been reported. ${ }^{22,23}$ More interestingly, the possibility of quantized thermal conductance at low temperatures is suggested. ${ }^{24}$

This work was supported in part by a Grant-in-Aid for Scientific Research from the Ministry of Education, Science and Culture of Japan (Grant No. 09640385). The work at Brown University was supported by the U.S. Department of Energy under Grant No. DE-FG02-86ER45267.
${ }^{1}$ See, for example, M. G. Cottam and D. R. Tilley, Introduction to Surface and Superlattice Excitations (Cambridge University Press, Cambridge, 1989); J. Sapriel and B. D. Rouhani, Surf. Sci. Rep. 10, 1189 (1989).

${ }^{2}$ T. Yao, Appl. Phys. Lett. 51, 1798 (1987).

${ }^{3}$ G. Chen, C. L. Tien, X. Wu, and J. S. Smith, J. Heat Transfer 116, 325 (1994).

${ }^{4}$ J. C. Cheng, F. H. Li, L. Guo, and S. Y. Zhang, Appl. Phys. A: Mater. Sci. Process. 61A, 441 (1995).

${ }^{5}$ X. Y. Yu, G. Chen, A. Verma, and J. S. Smith, Appl. Phys. Lett. 67, 3554 (1995).

${ }^{6}$ X. Y. Yu, L. Zhang, and G. Chen, Rev. Sci. Instrum. 67, 2312 (1996).

${ }^{7}$ G. Chen, J. Heat Transfer 119, 220 (1997).

${ }^{8}$ G. Chen and M. Neagu, Appl. Phys. Lett. 71, 2761 (1997).

${ }^{9}$ G. Chen, Phys. Rev. B 57, 14958 (1998).

${ }^{10}$ W. S. Capinski and H. J. Maris, Physica B 219\&220, 699 (1996).

${ }^{11}$ W. S. Capinski, M. Cardona, D. S. Katzer, H. J. Maris, K. Ploog, and T. Ruf, Physica B 263\&264, 530 (1999).

${ }^{12}$ W. S. Capinski, H. J. Maris, T. Ruf, M. Cardona, K. Ploog, and D. S. Katzer, Phys. Rev. B 59, 8105 (1999).
${ }^{13}$ S. M. Lee, D. G. Cahill, and R. Venkatasubramanian, Appl. Phys. Lett. 70, 2957 (1997).

${ }^{14}$ P. Hyldgaard and G. D. Mahan, Thermal Conductivity (Technomic, Lancaster, PA, 1996), Vol. 23, p. 172.

${ }^{15}$ P. Hyldgaard and G. D. Mahan, Phys. Rev. B 56, 10754 (1997).

${ }^{16}$ J. Piprek, T. Troger, B. Schroter, J. Kolodzey, and C. S. Ih, IEEE Photonics Technol. Lett. 10, 81 (1998).

${ }^{17}$ For the thermal conductivity in dielectric multilayered structures, see, for example, M. A. Weilert, M. E. Msall, J. P. Wolfe, and A. C. Anderson, Z. Phys. B 91, 179 (1993); M. A. Weilert, M. E. Msall, A. C. Anderson, and J. P. Wolfe, Phys. Rev. Lett. 71, 735 (1993).

${ }^{18}$ See, for example, A. A. Maradudin, P. A. Flinn, and R. A. Coldwell-Horsfall, Ann. Phys. (N.Y.) 15, 360 (1961).

${ }^{19}$ Y. Tanaka, M. Narita, and S. Tamura, J. Phys.: Condens. Matter 10, 8787 (1998).

${ }^{20}$ S. Y. Ren and J. D. Dow, Phys. Rev. B 25, 3750 (1982).

${ }^{21}$ S. Tamura, Phys. Rev. B 56, 12440 (1997); 30, 849 (1984).

${ }^{22}$ A. Balandin and K. L. Wang, Phys. Rev. B 58, 1544 (1998).

${ }^{23}$ S. G. Walkauskas, D. A. Broido, K. Kempa, and T. L. Reinecke, J. Appl. Phys. 85, 2579 (1999).

${ }^{24}$ L. G. C. Rego and G. Kirczenow, Phys. Rev. Lett. 81, 232 (1998). 